Mathematical Modelling AND ANALysis

Volume 17 Number 2, April 2012, 245-250

http://dx.doi.org/10.3846/13926292.2012.662532

(c) Vilnius Gediminas Technical University, 2012
Publisher: Taylor\&Francis and VGTU

http://www.tandfonline.com/TMMA

Print ISSN: 1392-6292

Online ISSN: 1648-3510

\title{
Zeros of the Lerch Transcendent Function*
}

\section{Ramūnas Garunkštis and Andrius Grigutis}

\author{
Vilnius University \\ Naugarduko 24, LT-03225 Vilnius, Lithuania \\ E-mail: ramunas.garunkstis@mif.vu.lt \\ E-mail(corresp.): andrius.grigutis@mif.stud.vu.lt
}

Received August 29, 2011; revised January 3, 2012; published online April 1, 2012

\begin{abstract}
We investigate the distribution of zeros of the Lerch transcendent function $\Phi(q, s, \alpha)=\sum_{n=0}^{\infty} q^{n}(n+\alpha)^{-s}$. We find an upper and lower estimates of zeros of the function $\Phi(q, s, \alpha)$ in any rectangle $\left\{s: \sigma_{1}<\operatorname{Re} s<\sigma_{2} \leq 1.73 \ldots, 0<\operatorname{Im} s \leq T\right\}$. Further we are interested in a computer calculations concerning the zeros of $\Phi(q, s, \alpha)$ in $\{s: \operatorname{Re} s>1,0<\operatorname{Im} s \leq 1000\}$.
\end{abstract}

Keywords: polylogarithm, Lerch transcendent, zero distribution.

AMS Subject Classification: 11M41.

\section{Introduction}

Let $s=\sigma+i t$ denotes a complex variable. The Lerch transcendent function is the analytic continuation of the series

$$
\Phi(q, s, \alpha)=\sum_{n=0}^{\infty} q^{n}(n+\alpha)^{-s}
$$

which converges for any real number $\alpha>0$ if $q$ and $s$ are complex numbers with either $|q|<1$, or $|q|=1$ and $\sigma>1$. Here we consider $\Phi(q, s, \alpha)$ as a function of $s$ with the parameters $q \in \mathbb{C}, 0<|q| \leq 1$, and $0<\alpha \leq 1$. Special cases include the Riemann zeta-function $\zeta(s)=\Phi(1, s, 1)$, the Hurwitz zeta-function $\zeta(s, \alpha)=\Phi(1, s, \alpha)$, the polylogarithm function $L i_{s}(q)=q \Phi(q, s, 1)$, and the Lerch zeta-function $L(\lambda, \alpha, s)=\Phi(\exp (2 \pi i \lambda), s, \alpha)$.

The Riemann zeta-function has no zeros in the right-half-plane $\sigma \geq 1$. In the left-half-plane $\sigma \leq 0$ it has only trivial zeros at even negative integers. The famous Riemann hypothesis $(\mathrm{RH})$ asserts that the remaining, nontrivial, zeros lie on the critical line $\sigma=1 / 2$.

The Hurwitz zeta-function $\zeta(s, \alpha)$ has infinitely many zeros in $1<\sigma<1+\alpha$ if $\alpha$ is transcendental or rational $\neq 1 / 2,1$ (Davenport and Heilbronn [2]). This

\footnotetext{
* Supported by grant No. MIP-94 from the Research Council of Lithuania.
} 
result was extended by Cassels [1] for $\alpha$ algebraic irrational. Let $1 / 2<\sigma_{1}<$ $\sigma_{2}<1$. Then Voronin [16] (for rational $\alpha \neq 1 / 2,1$ ) and Gonek [10] (for transcendental $\alpha$ ) proved that the number of zeros of $\zeta(s, \alpha)$ in the rectangle $\sigma_{1}<\sigma<\sigma_{2}, 0<t<T$ is approximately equal to $T$ for sufficiently large $T$. Gonek [11] also showed that for certain values of $\alpha$ the proportion of zeros of $\zeta(s, \alpha)$ on $\sigma=1 / 2$ is definitely less than 1 . In the complex $s$-plane trajectories of zeros $\rho=\rho(\alpha)$ of the Hurwitz zeta function were considered in [8] and [9]. Based on these trajectories, the classification of nontrivial zeros of the Riemann zeta function were introduced. For the zero distribution of the Lerch zetafunction see $[4,5,6,7,12]$.

Fornberg and Kölbig [3] investigated trajectories of zeros $\rho=\rho(q)$ of the polylogarithm function $L i_{s}(q)$ for real $q$ with $|q|<1$. They found that some trajectories tend towards the zeros of $\zeta(s)$ as $q \rightarrow-1$, and approach these zeros closely as $q \rightarrow 1-\delta$ for small but finite $\delta>0$. However, the later trajectories appear to descend to the point $s=1$ as $\delta \rightarrow 0$. Both, for $q \rightarrow-1$ and $q \rightarrow 1$, there are trajectories which do not tend towards zeros of $\zeta(s)$.

Next we consider the zeros of $\Phi(q, s, \alpha)$ for $0<\alpha<1$ and $q \in \mathbb{C}, 0<|q|<1$. Let $N_{\Phi}\left(\sigma_{1}, \sigma_{2}, T\right)=N_{\Phi}\left(\sigma_{1}, \sigma_{2}, T, q, \alpha\right)$ denote the number of zeros of $\Phi(q, s, \alpha)$ in the region $\left\{s: \sigma_{1}<\operatorname{Re} s<\sigma_{2}, 0<\operatorname{Im} s \leq T\right\}$. Let $\sigma_{0}=\sigma_{0}(q, \alpha)$ be a real number defined by the equality

$$
\sum_{n=1}^{\infty} \frac{|q|^{n}}{(n / \alpha+1)^{\sigma_{0}}}=1
$$

It is easy to see that $\sigma_{0} \leq c=1.73 \ldots$, where $\zeta(c)=\sum_{n=1}^{\infty} n^{-c}=2$, and that $\sigma_{0}$ can take any value between $-\infty$ and $c$.

Theorem 1. Let $q \in \mathbb{C}, 0<|q|<1$. Let $0<\alpha<1$ be a transcendental number. Then we have that, for any fixed strip $\sigma_{1}<\sigma<\sigma_{2} \leq \sigma_{0}$,

$$
T \ll N_{\Phi}\left(\sigma_{1}, \sigma_{2}, T\right) \ll T
$$

and $\Phi(q, s, \alpha)$ has no zeros for $\sigma>\sigma_{0}$.

The theorem is proved in Section 3.

Wiener and Wintner [17, Section 4] pointed to a possible relationship between the behaviour of the zeros in the right-half-plane $\sigma>1$ of the polylogarithm function and the Riemann Hypothesis. They proved that the Riemann Hypothesis is true if there exists a number $0<\varepsilon<1$ such that $\sum_{n=1}^{\infty} q^{n} n^{-s} \neq 0$ for $\sigma>1$ and $1-\varepsilon<q<1$. However, Montgomery [13] pointed that the polylogarithm function $L i_{s}\left(e^{-1 / N}\right)$ has zeros in the region $\sigma>1$ for all sufficiently large integers $N$, making Wiener and Winter theorem vacuous. Theorem 1 shows that the Lerch transcendent function $\Phi(q, s, \alpha)$ also has zeros in the region $\sigma>1$ for $0.92<q<1$ and transcendental $\alpha, 1 / 2<\alpha<1$. In the next section, we try to find explicit zeros in $\sigma>1$. We see that it is relatively easy to find zeros if $\alpha \neq 1$. In the case $\alpha=1$ the zeros in the right half-lane, $\sigma>1$ currently are out of reach. 


\section{Calculations}

The calculations of this section were done with programme MATHEMATICA. To calculate the number $N$ of zeros of $\Phi(q, s, \alpha)$ inside the contour $\Gamma$ we have used the well known formula

$$
N=\frac{1}{2 \pi i} \int_{\Gamma} \frac{(\Phi(q, s, \alpha))_{s}^{\prime}}{\Phi(q, s, \alpha)} d s .
$$

If the interior of the contour $\Gamma$ contains one zero $\rho$, then we find this zero using the following expression

$$
\rho=\frac{1}{2 \pi i} \int_{\Gamma} s \frac{(\Phi(q, s, \alpha))_{s}^{\prime}}{\Phi(q, s, \alpha)} d s .
$$

The zero $\rho$ can be adjusted by MATHEMATICA command FindRoot.

Let $R=\{s: \operatorname{Re} s>1,0<\operatorname{Im} s \leq 1000\}$. In Table 1, we present the number of zeros of function $\Phi(q, s, \alpha)$ for chosen $q$ and $\alpha$ in the region $R$. For example, we see that $\Phi(0.99, s, 0.9)$ has 34 zeros in $R$. In Table 1 , the last column describes zeros of the Hurwitz zeta-function, and the last row describes zeros of the polylogarithm function. In view of Montgomery's result [13] we expect that $\Phi(q, s, 1)$ has zeros in $\sigma>1$ for $q \geq 0.9$. If so, then Table 1 possibly indicates the different behaviour of zeros of $\Phi(q, s, \alpha)$ in $\sigma>1$ dependently on $\alpha=1$ or $\alpha \neq 1$.

Table 1. Number of zeros of the function $\Phi(q, s, \alpha)$ in the region $R$.

\begin{tabular}{|l|c|c|c|c|}
\hline$\alpha \backslash \mathrm{q}$ & $\mathbf{0 . 9}$ & $\mathbf{0 . 9 5}$ & $\mathbf{0 . 9 9}$ & $\mathbf{1}$ \\
\hline $\mathbf{0 . 9}$ & 2 & 8 & 34 & 40 \\
\hline $\mathbf{0 . 9 5}$ & 4 & 10 & 37 & 46 \\
\hline $\mathbf{0 . 9 9}$ & 14 & 27 & 41 & 45 \\
\hline $\mathbf{1}$ & 0 & 0 & 0 & 0 \\
\hline
\end{tabular}

In Table 2, we present zeros of functions $\Phi(0.9, s, 0.9), \Phi(0.9, s, 0.95)$, $\Phi(0.9, s, 0.99)$. In this table numbers were rounded up to two decimal places.

\section{Proof of Theorem 1}

First we formulate theorems of Kronecker and Rouché (see Tichmarsh [15, Section 8.3] and Tichmarsh [14, Section 3.42]).

Lemma 1 [Kronecker's theorem]. Let $a_{1}, a_{2}, \ldots, a_{N}$ be linearly independent real numbers, i.e. numbers such that relation $\lambda_{1} a_{1}+\cdots+\lambda_{N} a_{N}=0$ is possible only if $\lambda_{1}=\cdots=\lambda_{N}=0$. Let $b_{1}, \ldots, b_{N}$ be any real numbers, and $\varepsilon$ a given positive number. Then we can find a number $t$ and integers $x_{1}, \ldots, x_{N}$ such that $\left|t a_{n}-b_{n}-x_{n}\right|<\varepsilon, n=1, \ldots, N$. 
Table 2. Coordinates of zeros of the function $\Phi(q, s, \alpha)$ in the region $R$.

\begin{tabular}{rlll}
\hline & $\Phi(0.9, s, 0.9)$ & $\Phi(0.9, s, 0.95)$ & $\Phi(0.9, s, 0.99)$ \\
\hline 1 & $1.02+550.55 i$ & $1.07+108.39 i$ & $1.05+480.29 i$ \\
2 & $1.02+609.75 i$ & $1.01+135.21 i$ & $1.11+525.79 i$ \\
3 & - & $1.09+169.68 i$ & $1.08+588.57 i$ \\
4 & - & $1.07+196.67 i$ & $1.06+616.03 i$ \\
5 & - & - & $1.11+651.27 i$ \\
6 & - & - & $1.11+696.71 i$ \\
7 & - & - & $1.13+724.38 i$ \\
8 & - & - & $1.05+759.64 i$ \\
9 & - & - & $1.15+787.05 i$ \\
10 & - & - & $1.02+805.00 i$ \\
11 & - & - & $1.12+849.96 i$ \\
12 & - & - & $1.17+895.31 i$ \\
13 & - & - & $1.09+958.10 i$ \\
14 & - & & $1.00+985.50 i$ \\
\hline
\end{tabular}

Lemma 2 [Rouché's theorem]. Suppose that $f(s)$ and $g(s)$ are analytic functions inside and on a regular closed curve $\gamma$, and that $|f(s)|>|g(s)|$ for all $s \in \gamma$. Then $f(s)+g(s)$ and $f(s)$ have the same number of zeros inside $\gamma$.

The next lemma will be useful in the proof of Theorem 1.

Lemma 3. Let $q \in \mathbb{C}, 0<|q|<1$, and $0<\alpha<1$ be a transcendental number. Let $\sigma^{\prime}$ be a real number. Let $a(n)$ be a sequence of complex numbers such that $|a(n)|=1$. Let $\Phi_{a}(q, s, \alpha)=\sum_{n=0}^{\infty} a(n) q^{n}(n+\alpha)^{-s}$. Then for any $\varepsilon>0$ there exist $\tau \in \mathbb{R}$ such that

$$
\left|\Phi(q, s+i \tau, \alpha)-\Phi_{a}(q, s, \alpha)\right|<\varepsilon
$$

for $\operatorname{Re} s \geq \sigma^{\prime}$.

Proof. The Dirichlet series of the Lerch transcendent function converges absolutely for any $s$ if $|q|<1$. Therefore, for given $\sigma^{\prime}$ there is a positive integer $N$ such that, for any real number $u$ and $\sigma \geq \sigma^{\prime}$,

$$
\left|\sum_{n=N+1}^{\infty} \frac{q^{n}}{(n+\alpha)^{s+i u}}-\sum_{n=N+1}^{\infty} \frac{q^{n} a(n)}{(n+\alpha)^{s}}\right| \leq 2 \sum_{n=N+1}^{\infty} \frac{|q|^{n}}{(n+\alpha)^{\sigma}}<\frac{\varepsilon}{2} .
$$

Let $A=\sum_{n=0}^{N}|q|^{n} /(n+\alpha)^{\sigma^{\prime}}$. There is a sequence of real numbers $b(n)$ such that $e^{-2 \pi i b(n)}=a(n)$. The numbers $\log (n+\alpha)$ are linearly independent over $\mathbb{Q}$ since $\alpha$ is the transcendental number. By Kronecker's theorem (Lemma 1), there exist a real number $\tau$ and integers $x_{n}$ such that

$$
\left|\frac{\tau \log (n+\alpha)}{2 \pi}-b(n)-x_{n}\right|<\frac{\varepsilon}{8 \pi A} .
$$


In view of the inequality $\left|e^{z}-1\right| \leq 2|z|$, where $|z|<1$, we obtain

$$
\left|(n+\alpha)^{-i \tau}-a(n)\right|=\left|e^{-2 \pi i\left(\tau \log (n+\alpha) / 2 \pi-b(n)-x_{n}\right)}-1\right|<\frac{\varepsilon}{2 A} .
$$

By above we see that there is $\tau$ such that, for $\operatorname{Re} s \geq \sigma^{\prime}$,

$$
\left|\sum_{n=0}^{N} \frac{q^{n}}{(n+\alpha)^{s+i \tau}}-\sum_{n=0}^{N} \frac{q^{n} a(n)}{(n+\alpha)^{s}}\right| \leq \sum_{n=0}^{N} \frac{|q|^{n}}{(n+\alpha)^{\sigma^{\prime}}}\left|(n+\alpha)^{-i \tau}-a(n)\right|<\frac{\varepsilon}{2} .
$$

This and inequality (3.1) in view of triangle inequality, prove Lemma 3.

Proof of Theorem 1. For fixed $q$ and $\alpha$ the function $\Phi(q, s, \alpha)$ is bounded in any right half-plane, of complex numbers. This together with Theorem 9.62 of Titchmarsh [14] give the bound

$$
N_{\Phi}\left(\sigma_{1}, \sigma_{2}, T\right) \ll T .
$$

Further, if the strip $\sigma_{1}<\sigma<\sigma_{2}$ contains a zero of $\Phi(q, s, \alpha)$ then, arguing as in Lemma 1 of [4], we get the bound

$$
N_{\Phi}\left(\sigma_{1}, \sigma_{2}, T\right) \gg T \text {. }
$$

Next we will show that the function $\Phi(q, s, \alpha)$ has a zero in the strip $\sigma_{1}<$ $\sigma<\sigma_{2}$. We consider an auxiliary function $\Phi_{a}(q, \sigma, \alpha)=\sum_{n=0}^{\infty} a(n) q^{n}(n+\alpha)^{-\sigma}$. For fixed $\sigma, q$ and $\alpha$, let $V$ be a set of values taken by $\Phi_{a}(q, \sigma, \alpha)$ for independent $a(0), a(1), \ldots$, where $a(n) \in \mathbb{C}$ and $|a(n)|=1$. If $\sigma<\sigma_{0}$, then by Tichmarsh [15, Section 11.5, p. 297] we see that

$$
V=\left\{z:|z| \leq \sum_{n=0}^{\infty}|q|^{n}(n+\alpha)^{-\sigma}\right\} .
$$

Thus for $\sigma_{1}<\sigma^{\prime}<\sigma_{2}, q$, and $\alpha$ there is a sequence $a(1), a(2), \ldots$, such that $\Phi_{a}\left(q, \sigma^{\prime}, \alpha\right)=0$.

Let $0<\varepsilon^{\prime}<\min \left(\sigma^{\prime}-\sigma_{1}, \sigma_{2}-\sigma^{\prime}\right)$ be such that $\Phi_{a}(q, s, \alpha) \neq 0$ for $\left|s-\sigma^{\prime}\right|=\varepsilon^{\prime}$. Let

$$
\varepsilon=\min _{\left|s-\sigma^{\prime}\right|=\varepsilon^{\prime}}\left|\Phi_{a}(q, s, \alpha)\right| .
$$

By Lemma 3 there is a real shift $\tau$ such that

$$
\left|\Phi(q, s+i \tau, \alpha)-\Phi_{a}(q, s, \alpha)\right|<\varepsilon
$$

for $\operatorname{Re} s \geq \sigma_{1}$. Hence Rouché's theorem gives that $\Phi(q, s, \alpha)$ has a zero in the disk $\left|s-\sigma^{\prime}-i \tau\right|<\varepsilon^{\prime}$, which is contained in the strip $\sigma_{1}<\sigma<\sigma_{2}$. By this Theorem 1 is proved.

\section{Conclusions}

Let $0<q<1$ and $1 / 2<\alpha \leq 1$. We expect that the Lerch transcendent function $\Phi(q, s, \alpha)$ has zeros in $\operatorname{Re} s>1$, if $q$ is sufficiently near to 1 . For $\alpha=1$ this is due to Montgomery [13]. Here we prove the case when $\alpha$ is a transcendental number. However, computer calculations indicate the different behaviour of zeros of $\Phi(q, s, \alpha)$ in $\operatorname{Re} s>1$ dependently on $\alpha=1$ or $\alpha \neq 1$. 


\section{References}

[1] J.W.S. Cassels. Footnote to a note of Davenport and Heillbronn. J. London Math. Soc., 36:177-184, 1961. http://dx.doi.org/10.1112/jlms/s1-36.1.177.

[2] H. Davenport and H. Heillbronn. On the zeros of certain Dirichlet series I, II. J. London Math. Soc., 11:111-115, 307-312, 1936.

[3] B. Fornberg and K.S. Kölbig. Complex zeros of the Jonquiere or polylogarithm function. Math. Comput., 29:582-599, 1975.

[4] R. Garunkštis. On zeros of the Lerch zeta-function. II. In B. Grigelionis et al.(Ed.), Probability Theory and Mathematical Statistics, Proceedings of the Seventh Vilnius Conf. 1998, pp. 267-276. TEV/Vilnius, VSP/Utrecht, 1999.

[5] R. Garunkštis. On zeros of the Lerch zeta-function. III. Liet. Matem. Rink., supl.:24-30, 1999.

[6] R. Garunkštis and A. Laurinčikas. On zeros of the Lerch zeta-function. In S. Kanemitsu and K. Györy(Eds.), Number Theory and Its Applications, pp. 129-143. Kluwer Academic Publishers, 1999.

[7] R. Garunkštis and J. Steuding. On the zero distributions of Lerch zeta-functions. Analysis, 22:1-12, 2002.

[8] R. Garunkštis and J. Steuding. On the distribution of zeros of the Hurwitz zetafunction. Math. Comput., 76:323-337, 2007. http://dx.doi.org/10.1090/S0025-5718-06-01882-5.

[9] R. Garunkštis and J. Steuding. Questions around the nontrivial zeros of the Riemann zeta-function - computations and classifications. Math. Model. Anal., 16(1):72-81, 2011. http://dx.doi.org/10.3846/13926292.2011.560616.

[10] S.M. Gonek. Analytic properties of zeta and L-functions. PhD thesis, University of Michigan, 1979.

[11] S.M. Gonek. The zeros of Hurwitz's zeta-function on $\sigma=1 / 2$. In Analytic Number Theory, Proc. Conf., Temple Univ./Phila. 1980, volume 899 of Lect. Notes Math., pp. 129-140, 1981.

[12] A. Laurinčikas and R. Garunkštis. The Lerch Zeta-Function. Kluwer Academic Publishers, 2002.

[13] H.L. Montgomery. Zeros of approximations to the zeta function. In Stud. Pure Math., pp. 497-506. Birkhäuser, Basel, 1983.

[14] E.C. Titchmarsh. The Theory of Functions. Oxford, 1939.

[15] E.C. Titchmarsh. The Theory of the Riemann Zeta-Function. Oxford, 1951.

[16] S.M. Voronin. Analytical properties of the Dirichlet generating series of arithmetical objects. PhD thesis, Moskow, 1977. (in Russian)

[17] N. Wiener and A. Wintner. Notes on Pólya's and Turán's hypotheses concerning Liouville's factor. Rend. Circ. Mat. Palermo, 6(2):240-248, 1957. http://dx.doi.org/10.1007/BF02843849. 